\title{
Detección de genes de virulencia en cepas de Enterococcus faecalis susceptibles y resistentes a aminoglucósidos
}

\author{
Juan Silva, Yara Rodríguez, Jorge Araya, Joselyne Gahona, Nicomedes Valenzuela, \\ Katheryne Guerrero, John Báez, Fernando Baquero y Rosa del Campo
}

\section{Detection of virulence genes in aminoglycoside susceptible and resistant Enterococcus faecalis}

Background: Enterococcus spp. is an important cause of nosocomial infections A number of virulence factors that may enhance its ability to colonize have been described. Enterococcus is capable of acquiring resistance genes, including high-level resistance (HLR) to aminoglycoside antibiotics. Aim: to investigate the prevalence of genes encoding virulence factors in aminoglycosides susceptible and resistant $E$. faecalis. Materials and Methods: A total of 80 E. faecalis isolates from clinical (n: 52) and poultry samples (n: 28) were included in this study. Bacterial identification was performed by biochemical tests and phenotypificationwas done using the Phene-Plate ${ }^{\mathrm{TM}}$ sys- $^{-}$ tem. Susceptibility to different antimicrobial agents was determined by the agar dilution method. Virulence genes ace I, agg, gelE and efaA were detected by multiplex PCR. Results: All isolates were susceptible to vancomycin and ampicillin. HLR to gentamicin (13.5\%) and streptomycin (9.6\%) was detected only in clinical isolates. The phenotyping revealed a great diversity of PhP-types, but only one clone with 7 strains of similar characteristics was found. The efaA gen was detected in $100 \%$ of the isolates. aceI gene was present in $94.2 \%$ and $75 \%$, agg gene in $73.1 \%$, and $67.9 \%$, and gelE gene in $57.5 \%$ and $28.6 \%$ of the clinical and chicken isolates, respectively. Only 6 strains with HLR to aminoglycosides, belonging to the same phenotype, had the acel, agg, gelE and efaA genes. Conclusions: E. faecalis with virulence genes and HLR to aminoglycosides were isolated from clinical and chicken samples in Antofagasta. More studies will be necessary to establish an association.

Key words: High levels of resistance, virulence genes, antibiotics, aminoglycosides, Enterococcus.

Palabras clave: Resistencia de alto nivel a aminoglucósidos, genes de virulencia, antimicrobianos, Enterococcus.

\section{Introducción}

$\mathrm{E}$ 1 género Enterococcus forma parte de la microbiota gastrointestinal del hombre y de los animales. Durante mucho tiempo no fueron considerados como patógenos primarios. Sin embargo, cepas de Enterococcus spp resistentes a vancomicina (ERV) han emergido como una causa importante de infecciones nosocomiales en todo el mundo ${ }^{1,2}$. Este incremento como agente de infecciones nosocomiales se debe, en parte, a su capacidad de adquirir resistencia a los antimicrobianos, lo que hace difícil el tratamiento y también, a su capacidad de adaptarse fácilmente a los cambios del medio. En Estados Unidos de América se aíslan cepas ERV en $25 \%$ de las infecciones nosocomiales en las Unidades de Cuidados Intensivos (UCI) y están considerados como uno de los tres patógenos más comúnmente aislados en las infecciones nosocomiales ${ }^{3}$.

Enterococcus spp representan un gran desafío terapéutico debido a su resistencia intrínseca a varios antimicrobianos. Ampicilina y vancomicina son los antimicrobianos usados normalmente en el tratamiento de las infecciones producidas por este agente ${ }^{4}$. Además de la resistencia intrínseca, poseen capacidad de adquirir genes de resistencia y de virulencia, que son transferidos por plasmidios o transposones conjugativos. Una gran importancia ha sido reconocida para las cepas de Enterococcus que adquieren resistencia de alto nivel (RAN) a aminoglucósidos ${ }^{5,6}$.

A pesar de su patogenicidad relativa, varios factores de virulencia han sido descritos en este género, codificados en genes de virulencia, mayormente en cepas de Enterococcus faecalis ${ }^{7}$. Entre ellos, destacan el antígeno A (efaA), la sustancia de agregación (asaI o agg), gelatinasa ( gelE), proteína enterocócica de superficie (esp), citolisina (cylA), adhesina del colágeno (aceI) y hialuronidasa $(h y l)^{7-13}$.

El gen asaI o agg, albergado en un plasmidio, codifica para la producción de una sustancia de agregación (feromona inducible) que participa en la conjugación de enterococos ${ }^{7}$. La sustancia de agregación aumenta la adherencia a las células de los túbulos renales, a las células
Universidad de Antofagasta, Antofagasta, Chile.

Departamento de Tecnología Médica (JSA, YRG, JAR, JGR, NVL, KGO, JBV).

Hospital Universitario Ramón y Cajal, Madrid, España Servicio de Microbiología (FB, RDC).

Los autores declaran no tener conflicto de intereses

Financiado por Proyecto PROIM No 1357, Dirección de Investigación de la Universidad de Antofagasta y Proyecto $N^{\circ} 4549$ Minera Escondida, Antofagasta, Chile.

Recibido: 28 de marzo de 2012 Aceptado: 9 de diciembre de 2012

Correspondencia a:

Juan Silva Avalos jsilva@uantof.cl 
intestinales y finalmente, se ha asociado a endocarditis en modelos animales ${ }^{8}$.

El gen cromosomal gelE codifica para una gelatinasa, que es una metalopeptidasa extracelular que hidroliza colágeno, gelatina, caseína, hemoglobina y pequeños péptidos y se ha visto que produce exacerbación de la endocarditis en modelos animales 9 .

El gen aceI codifica para una adhesina que es capaz de interactuar con el colágeno tipo I y IV y la laminina, favoreciendo la unión de enterococos a las células del hospedero ${ }^{13}$.

En estudios anteriores, hemos aislados cepas de $E$. faecalis de origen clínico y ambiental, las cuales presentaban ausencia de resistencia a vancomicina, pero exhibían resistencia de alto nivel a aminoglucósidos ${ }^{14,15}$. Este trabajo tiene como objetivo estudiar la presencia de genes de virulencia en diferentes fenotipos bioquímicos de E. faecalis susceptibles y resistentes a aminoglucósidos.

\section{Material y Métodos}

\section{Cepas bacterianas}

Se incluyeron 80 cepas de E. faecalis, 52 aisladas de muestras clínicas en el Hospital Regional de Antofagasta: orina (n: 29), herida operatoria (n: 8), sangre (n: 1), secreción vaginal (n: 10), otras muestras clínicas (n: 4); y cepas provenientes de pollos expendidos en locales comerciales (n: 28), todas recolectadas en el año 2008. Las cepas fueron identificadas mediante pruebas bioquímicas con diferentes sustratos, de acuerdo a lo descrito por Manero y Blanch ${ }^{16}$ También, se identificaron por medio de la comparación de los resultados del Phene-Plate ${ }^{R}$ con datos de cepas referenciales del patrón de PhP del Programa PhPWiM4 ${ }^{17}$.

\section{Susceptibilidad in vitro a antimicrobianos}

Se evaluó la concentración inhibitoria mínima (CIM) de las cepas de $E$. faecalis por técnica de dilución en placa,

\section{Tabla 1. Oligonucleótidos partidores y productos en la detección de los genes de} virulencia en cepas de $E$. faecalis por RPC

\begin{tabular}{|c|c|c|c|}
\hline $\begin{array}{l}\text { Genes de } \\
\text { virulencia }\end{array}$ & $\begin{array}{l}\text { Secuencia nucleotídica } \\
\qquad\left(5^{\prime}-3^{\prime}\right)\end{array}$ & $\begin{array}{l}\text { Tamaño de los amplicones } \\
\text { (pb) }\end{array}$ & Referencia \\
\hline ace & $\begin{array}{c}\text { GTCGTCTTTTCACTTGTTTCT } \\
\text { GAGCAAAAGTTCAATCGTTGAC }\end{array}$ & 1.003 & 22 \\
\hline $\operatorname{aggA}$ & $\begin{array}{l}\text { AAGAAAAAGAAGTAGACCAAC } \\
\text { AAACGGCAAGACAAGTAAATA }\end{array}$ & 1.553 & 8 \\
\hline efaA & $\begin{array}{l}\text { GACAGACCCTCACGAATA } \\
\text { AGTTCATCATGCTGTAGTA }\end{array}$ & 705 & 8 \\
\hline gelE & $\begin{array}{l}\text { ACGCATTGCTTTTCCATC } \\
\text { ACCCCGTATCATTGGTTT }\end{array}$ & 419 & 8 \\
\hline
\end{tabular}

de acuerdo a los criterios establecidos por el CLSI ${ }^{18}$. Se incluyeron los siguientes antimicrobianos: amikacina (AMK), ampicilina (AMP), penicilina (P), vancomicina $(\mathrm{V})$, gentamicina $(\mathrm{G})$, ciprofloxacina (CIP), tetraciclina (T), eritromicina (E), estreptomicina (SM) y cloranfenicol (CAF). Los resultados fueron expresados como la CIM $_{50}$ $\mathrm{y} \mathrm{CIM}_{90}$, y se determinaron los porcentajes de resistencia. Además, se determinó la resistencia de alto nivel (RAN) a aminoglucósidos, los que correspondieron a $>500 \mu \mathrm{g} / \mathrm{ml}$ para $\mathrm{G}$ y $>2.000 \mu \mathrm{g} / \mathrm{ml}$ para SM. En todos los ensayos, se incluyó la cepa de E. faecalis ATCC 29212, como control.

\section{Tipificación de fenotipos bioquímicos}

La determinación de los fenotipos bioquímicos (biochemical fingerprinting) se realizó por el método de $\mathrm{PhP}$ RF (Phene-Plate Techniques AB, Sweden), de acuerdo a recomendaciones descritas en trabajos anteriores ${ }^{14}$. A partir de una colonia de Enterococcus sp cultivada en placa de agar sangre se sembró un tubo con $10 \mathrm{ml}$ de proteosa peptona (Difco) $0,1 \%$, conteniendo azul de bromotimol $0,01 \%$ y se inocularon alícuotas de $150 \mu \mathrm{l}$ a los pocillos de microplacas que contenían 8 sets con 11 sustratos deshidratados seleccionados para identificar los fenotipos bioquímicos de Enterococcus spp. Las microplacas se incubaron a $37^{\circ} \mathrm{C}$ hasta un total de $64 \mathrm{~h}$. La cinética de las reacciones fue evaluada midiendo los valores de absorbancia a las 16, 40 y 64 h. El fenotipo bioquímico (fingerprinting) se calculó como el valor promedio para cada reactivo de las tres lecturas, mediante el programa computacional PhP-WiM4. Las similitudes entre las cepas ensayadas fueron calculadas como coeficientes de correlación $(r)$ y fueron reunidas de acuerdo al método de grupo par no pesados con promedios aritméticos (UPGMA) ${ }^{17}$. Los fenotipos bioquímicos que se encontraron en más de una cepa fueron llamados fenotipos comunes ( $\mathrm{PhP}-\mathrm{C}$ ), $\mathrm{y}$ aquellos que presentaron solamente un aislado fueron llamados fenotipos solos (PhP-S).

\section{Detección de genes de virulencia}

La amplificación de los genes de virulencia se realizó por técnica de RPC múltiple de los genes aceI, gelE, agg y efa $\mathrm{A}^{7}$. Los partidores utilizados fueron descritos previamente $^{7,13}$. La secuencia de los partidores y tamaño esperado de los amplicones para cada ensayo de RPC se muestran en la Tabla 1. En los ensayos se incluyó, además, una cepa de E. raffinosus y una de E. gallinarum como controles negativos. Las cepas de Enterococcus se sembraron en agar sangre a $37^{\circ} \mathrm{C}$ por $18-24 \mathrm{~h}$. Se tomaron varias colonias y se hizo una emulsión espesa en microtubos que contenían $200 \mu$ l de agua destilada estéril, que se pusieron a ebullición $\left(100{ }^{\circ} \mathrm{C}\right)$ durante $10 \mathrm{~min}$, luego se centrifugó a $12.000 \mathrm{rpm}$ por $5 \mathrm{~min}$. Se tomaron $2 \mu \mathrm{ldel}$ sobrenadante y se agregó a la mezcla de reacción, en un volumen final de $25 \mu \mathrm{l}$, conteniendo: $1 \mu \mathrm{IgCl}_{2} 50 \mathrm{mM}$, 
2,5 $\mu 1$ tampón RPC, $1 \mu \mathrm{l}$ dNTPs 10 mM, 1 ml de cada partidor 100 pmol, 1 U Taq polimerasa (promega) y 13,5 $\mu 1$ agua milliQ ultrapura. El primero de los sets contenía los partidores de los genes ace I y agg y el segundo set efaA y gelE. La amplificación de la mezcla se hizo en un equipo de RPC (PTC-100 Programable Termal Controller, MJ Research, Inc., Watertonw, MA), con una denaturación inicial de $95^{\circ} \mathrm{C}$ por $5 \mathrm{~min}$; seguido de 35 ciclos de $95^{\circ} \mathrm{C}$ por $30 \mathrm{seg}, 56^{\circ} \mathrm{C}$ por $1 \mathrm{~min}$ y $72^{\circ} \mathrm{C}$ por $2 \mathrm{~min}$; y una extensión final $72^{\circ} \mathrm{C}$ por $10 \mathrm{~min}$. Después de la amplificación, una muestra de $6 \mu \mathrm{L}$ de cada reacción fue analizada mediante electroforesis en un gel de agarosa a $1 \%$ con bromuro de etidio (Equipo Power Pac Basic, BioRad, USA). Se usó como marcador de tamaño molecular de ADN un 100-bp DNA ladder (Invitrogen, Merelbeke, Belgium).

\section{Análisis estadístico}

La comparación de los datos de las cepas de Enterococcus recuperadas de muestras clínicas y de pollos se hizo mediante el análisis de las variables cualitativas con el test de Fisher, utilizando el Programa Minitab 15. Un valor $\mathrm{p}<0,05$ fue considerado estadísticamente significativo.

\section{Resultados}

Los resultados de las pruebas bioquímicas permitieron la identificación de las cepas de E. faecalis y los análisis del Ph-plate confirmaron estos resultados. En la Tabla 2 se muestra la gran susceptibilidad que exhibieron las cepas de Enterococcus frente a V y AMP, con una $\mathrm{CIM}_{50}$ y $\mathrm{CIM}_{90}$ que fluctuaron entre 0,5 y $4 \mu \mathrm{g} / \mathrm{ml}$. Se observó resistencia a CAF, 90,9 y 39,3\% (p<0,05); T 57,6 y $35,7 \%$ (p > 0,05); E 32,7 y 14,3\% (p>0,05) de las cepas clínicas y de pollo, respectivamente. Un 30\% de las cepas clínicas exhibió resistencia a CIP $(\mathrm{p}<0,05)$. En general, las cepas clínicas fueron más resistentes a los antimicrobianos, pero en algunos casos esta diferencia no fue estadísticamente significativa. Las diferencias entre las cepas de Enterococcus aisladas de muestras clínicas y de pollos con RAN a G 13,5/3,6\% (p 0,250), RAN a AMK $11,5 / 3,6 \%$ (p 0,412), y RAN a SM 9,6/0\% (p 0,156), no son estadísticamente significativas.

El sistema Phene-Plate permitió la identificación de 16 fenotipos bioquímicos diferentes que no presentaron correlación alguna con el origen de aislado, solamente el PhP-tipo C3 fue el más frecuentemente aislado en las cepas clínicas ( 7 cepas) y el PhP-tipo C2 en las de pollo.

Los resultados de la RPC múltiple mostraron que los amplicones obtenidos correspondieron a los tamaños moleculares para cada uno de los genes de virulencia ensayados. En la Figura 1, la electroforesis muestra una banda que corresponde al gen efaA (705 pb) presente en todas las cepas de E. faecalis y ausente en E. raffinosus y E. gallinarum. También se aprecia otra banda de 419 bp correspondiente al gelE presente en varias cepas de E. faecalis. En la Figura 2, las bandas corresponden a los genes $\operatorname{agg}(1.553 \mathrm{pb})$ que se encontraba presente en varias cepas de E. faecalis y también se aprecia una banda de $1.003 \mathrm{bp}$, correspondiente al gen aceI que se detectó en algunas de las cepas ensayadas.

Tabla 2. Distribución de la $\mathrm{CIM}_{50}$ y $\mathrm{CIM}_{90}$ de cepas de $E$. faecalis a antimicrobianos

\begin{tabular}{|c|c|c|c|c|c|c|c|}
\hline \multirow[t]{2}{*}{ Antimicrobianos } & \multirow{2}{*}{$\begin{array}{c}\text { Rango } \\
\text { CIM } \mu \mathrm{g} / \mathrm{ml}\end{array}$} & \multicolumn{3}{|c|}{ Cepas clínicas } & \multicolumn{3}{|c|}{ Cepas de pollos } \\
\hline & & $\mathrm{CIM}_{50}$ & $\mathrm{CIM}_{90}$ & $\% R$ & $\mathrm{CIM}_{50}$ & $\mathrm{CIM}_{90}$ & $\% \mathbf{R}$ \\
\hline Amikacina $^{1}$ & $4-2.024$ & 8 & 1.024 & $11,5^{*}$ & 4 & 8 & $3,6^{*}$ \\
\hline Ampicilina & $0,25-8$ & 1 & 4 & 0 & 0,5 & 1 & 0 \\
\hline Ciprofloxacina ${ }^{1}$ & $0,25-64$ & 0,5 & 8 & 30,8 & 0,5 & 0,5 & 0 \\
\hline Cloranfenicol' ${ }^{1}$ & $2-256$ & 64 & 128 & 90,9 & 16 & 32 & 39,3 \\
\hline Eritromicina $^{2}$ & $0,5-16$ & 1 & 8 & 32,7 & 0,5 & 4 & 14,3 \\
\hline Estreptomicina ${ }^{2}$ & $4-2.048$ & 32 & 1.024 & $9,6^{*}$ & 32 & 64 & 0 \\
\hline Gentamicina $^{2}$ & $4-2.048$ & 8 & 1.024 & $13,5^{*}$ & 4 & 32 & $3,6^{*}$ \\
\hline Penicilina & $0,25-2$ & 0,5 & 2 & 0 & 0,5 & 1 & 0 \\
\hline Tetraciclina $^{2}$ & $2-128$ & 16 & 128 & 57,6 & 0,5 & 64 & 35,7 \\
\hline Vancomicina & $0,25-8$ & 0,5 & 2 & 0 & 0,5 & 1 & 0 \\
\hline
\end{tabular}

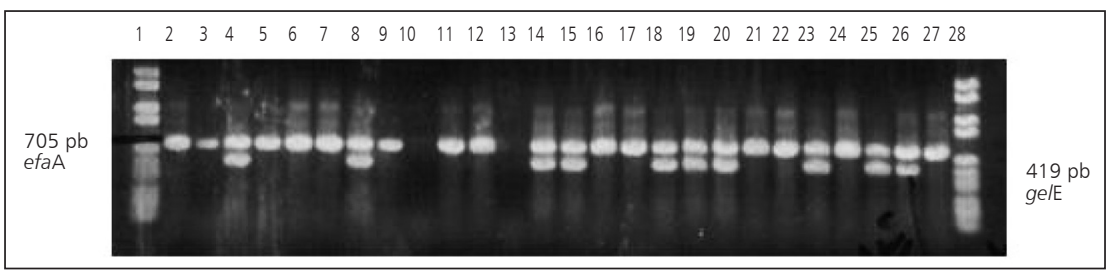

Figura 1. Línea 1 y 28 marcador de tamaño molecular (100bp). Líneas 2, 3, 4, 5, 6, 7, 8, 9, 11, 12 , $14,15,16,17,18,19,20,21,22$ y 23 cepas clínicas $E$. faecalis y líneas 24, 25, 26 y 27 cepas de $E$. faecalis de pollos fueron positivas para el gen efaA. Línea 10 E. raffinosus y línea 13 E. gallinarum fueron negativas para el gen efaA. Las bandas inferiores corresponden al gen ge/E, el cual detectó en las líneas 4, 8, 14, 15, 18, 19, 20 y 23 de las cepas clínicas de $E$. faecalis y línea 25 y 26 de las cepas de pollos.

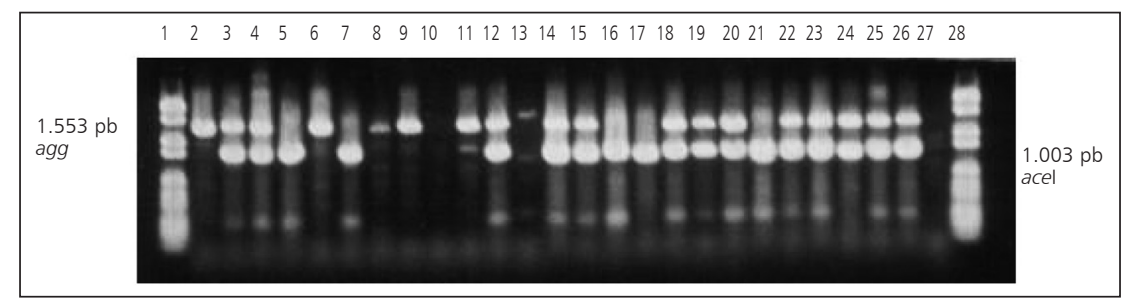

Figura 2. Línea 1 y 28 marcador de tamaño molecular (100bp). Líneas 2, 3, 4, 6, 8, 9, 11, 12, 14 , $15,18,19,20,21,22$ y 23 cepas clínicas $E$. faecalis y líneas 24,25 y 26 de cepas de $E$. faecalis de pollos fueron positivas para el gen agg. Línea 10 E. raffinosus y línea 13 E. gallinarum fueron negativas para el gen agg. Las bandas inferiores corresponden al gen acel, el cual detectó en las líneas 3, 4, $5,7,12,14,15,16,17,18,19,20,21,22$ y 23 de las cepas clínicas de $E$. faecalis y línea 24,25 y 26 de las cepas de pollos. 


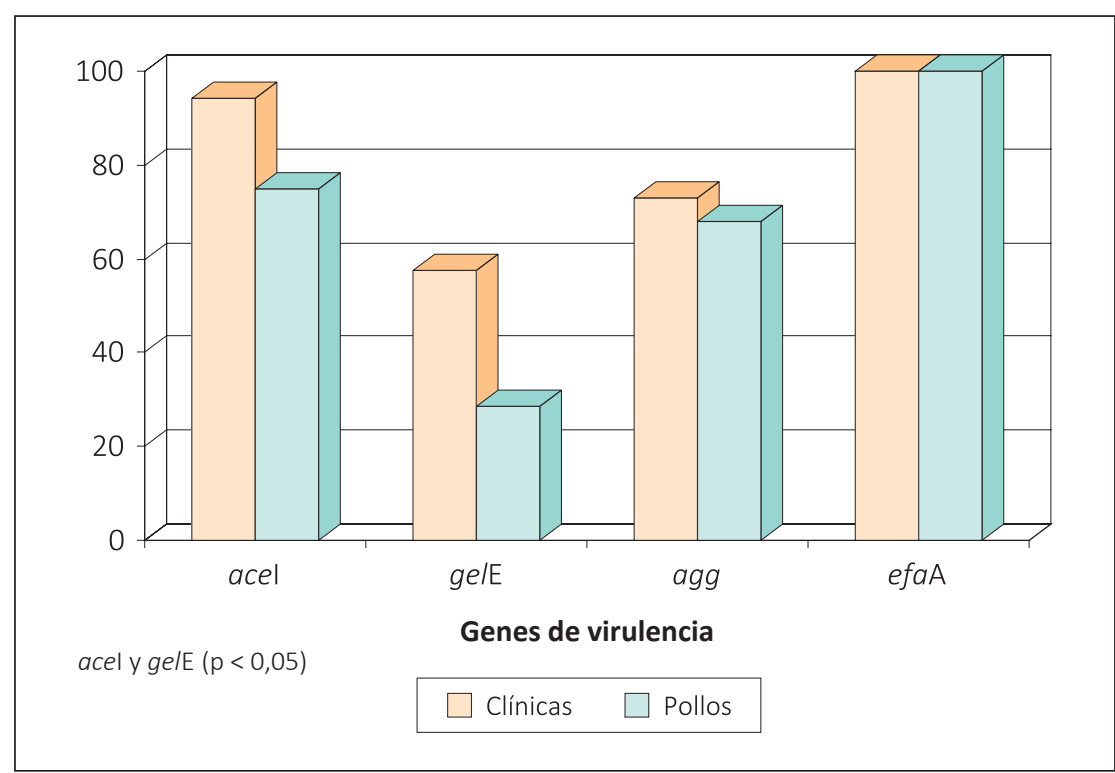

Figura 3. Porcentajes de los genes de virulencia presentes en cepas de Enterococcus faecalis aisladas de muestras clínicas y provenientes de pollos. Antofagasta, Chile.

Tabla 3. Combinaciones de los genes acel, ge/E, agg y efaA presentes en cepas de Enterococcus faecalis de origen clínico y avícola aisladas en Antofagasta, Chile

\begin{tabular}{lcccccc} 
& \multicolumn{3}{c}{ Cepas bacterianas } & \multicolumn{4}{c}{ Genes de virulencia } \\
Origen & $\mathbf{n}$ & $\%$ & acel & ge/E & agg & efaA \\
Clínicas (n: 52) & 30 & $57,7^{*}$ & + & + & + & + \\
& 8 & 15,4 & + & - & + & + \\
& 11 & 21,1 & + & - & - & + \\
Pollos (n: 28) & 4 & 7,7 & - & - & - & + \\
& 5 & $17,9^{*}$ & + & + & + & + \\
& 9 & 32,1 & + & - & + & + \\
& 3 & 10,7 & - & + & + & + \\
& 7 & 25,0 & + & - & - & + \\
$*(p<0,05)$. & 4 & 14,3 & - & - & + & + \\
\hline
\end{tabular}

En la Figura 3, se muestran los porcentajes de los genes de virulencia encontrados en las distintas cepas de Enterococcus. Estos revelan que el gen efaA, que codifica para un antígeno de superficie, estaba presente en $100 \%$ de cepas de E. faecalis, tanto en las de origen clínico como en las aisladas de pollos. El gen efaA no se detectó en las cepas de E. raffinosus y E. gallinarum. El gen aceI (adhesina), estaba presente en $94,2 \%$ de las cepas clínicas y en $75 \%$ de las provenientes de pollos (p 0,028 ). El gen que codifica para la sustancia de agregación $(\mathrm{agg})$ fue encontrado en $73,1 \%$ de las cepas clínicas y $67,9 \%$ en las obtenidas de pollos (p 0,616). Finalmente, el gen gelE fue encontrado en menor proporción con $57,7 \%$ en las cepas clínicas y $28,6 \%$ en las aisladas de pollos (p 0,019). En general se aprecia que los genes de virulencia están presentes en mayor proporción en las cepas de E. faecalis que fueron aisladas de muestras clínicas, pero esta diferencia es estadísticamente significativa sólo para los genes aceI y gelE.

En la Tabla 3, se presenta la combinación de los genes aceI, gelE, agg y efaA presentes en las cepas de E. faecalis. Se encontró que los cuatro genes estaban presentes en $57,7 \%$ de las cepas clínicas y en menor proporción en las recuperadas de pollos, alcanzando a $17,9 \%(\mathrm{p}<0,05)$. Las otras combinaciones de tres y dos genes de virulencia juntos, se encontraron en porcentajes variables en los aislados clínicos y procedentes de pollos.

En la Tabla 4, se exhiben los resultados de los análisis de RPC que mostraron la presencia de genes de virulencia en la mayoría de las cepas de E. faecalis, los que se encontraron en mayor proporción en las cepas resistentes a altos niveles de aminoglucósidos.

\section{Discusión}

En los últimos años, E. faecalis resistente a antimicrobianos ha emergido como un importante patógeno

Tabla 4. Distribución de los genes de virulencia y PhP-tipo en cepas de E. faecalis resistentes a altos niveles (RAN) a aminoglucósidos

\begin{tabular}{|c|c|c|c|c|c|c|c|c|c|c|c|}
\hline \multirow[t]{2}{*}{ n de cepa } & \multicolumn{4}{|c|}{ Genes de virulencia } & \multirow{2}{*}{$\begin{array}{l}\text { PhP } \\
\text { Tipo }\end{array}$} & \multirow{2}{*}{$\begin{array}{c}\text { Origen } \\
\text { de cepas }\end{array}$} & \multicolumn{5}{|c|}{$\mathrm{CIM}(\mu \mathrm{g} / \mathrm{ml})$} \\
\hline & acel & $g e / \mathbb{E}$ & agg & efaA & & & AM & V & AK & G & ES \\
\hline$E-34$ & - & - & + & + & $\mathrm{C} 2$ & P & 1 & 0,5 & 1.024 & 2.048 & 64 \\
\hline $\mathrm{E}-76$ & + & + & + & + & C3 & C & 0,5 & 0,25 & 1.024 & 1.024 & 1.024 \\
\hline E-77 & + & + & + & + & C3 & $C$ & 0,5 & 0,25 & 1.024 & 1.024 & 1.024 \\
\hline $\mathrm{E}-91$ & + & + & + & + & C3 & C & 1 & 0,25 & 1.024 & 2.048 & 128 \\
\hline E-99 & + & - & + & + & C3 & $C$ & 0,5 & 0,25 & 1.024 & 1.024 & 128 \\
\hline E-103 & + & + & + & + & C3 & $C$ & 1 & 0,25 & 1.024 & 1.024 & 2.048 \\
\hline E-105 & + & + & + & + & C3 & C & 1 & 0,25 & 2.048 & 2.048 & 2.048 \\
\hline $\mathrm{E}-117$ & + & + & + & + & C3 & C & 1 & 0,5 & 1.024 & 1.024 & 2.048 \\
\hline
\end{tabular}


nosocomial y también de infecciones adquiridas en la comunidad, cuya evolución y diseminación de resistencia ha sido facilitada por el uso de antimicrobianos a nivel hospitalario y en la comunidad, como así también, por el uso como aditivo de la alimentación en la crianza de animales $^{19}$. En Chile, los reservorios y fuentes de E. faecalis resistentes a antimicrobianos no han sido completamente definidos e investigados, como así también, los mecanismos que ponen en juego para evadir la acción de los antimicrobianos y de sus capacidades de producir daño.

En relación a la resistencia a antimicrobianos, destaca la ausencia de resistencia a $\mathrm{V}$, hallazgo similar a lo descrito en otros trabajos ${ }^{15}$. Esta situación puede deberse al uso restringido de $\mathrm{V}$ y por otro lado, su uso en el Hospital Regional de Antofagasta está orientado al tratamiento de los pacientes con condiciones de gravedad. Sin embargo, hoy día el aislamiento de ERV va en aumento en todo el país ${ }^{20-23}$.

En años recientes, se ha detectado un aumento de la prevalencia de resistencia a $\mathrm{T}$, como así también a $\mathrm{CIP}^{23}$. En nuestro estudio, más de $58 \%$ de las cepas de $E$. faecalis presentaban resistencia a T. Frente a CIP se encontró una resistencia de $30,8 \%$, fenómeno presente sólo en cepas de origen clínico.

La RAN a G se encontró en 13,5\%, a AMK 11,5\% y a $\mathrm{SM}$ en $9,6 \%$ de las cepas de origen clínico. Si bien estos resultados no son elevados, ya son una realidad en nuestro medio. Respecto a esto, en una publicación anterior se encontró $10 \%$ de cepas de Enterococcus spp con RAN a G en los aislados de cinco hospitales del norte de Chile $^{15}$. Estos resultados señalan la posible presencia de enzimas modificantes de aminoglucósidos en estas cepas, especialmente la enzima bifuncional que codifica el gen aac6'-aph2" y que ha sido descrita en E. faecalis ${ }^{24,25}$. El aislamiento de cepas de Enterococcus con RAN a G podría ir en aumento en los hospitales del norte de Chile, lo que constituiría un serio problema, pues disminuye la posibilidad de su asociación con $\beta$-lactámicos o $\mathrm{V}$ para obtener un efecto sinérgico y tratar en mejor forma las infecciones graves ${ }^{4,5}$.

Los resultados de la tipificación de las cepas de Enterococcus por el sistema Ph-Plate, nos mostraron la existencia una gran diversidad de fenotipos, tanto en las cepas clínicas como en las aisladas de pollo. Sin embargo, se encontraron algunos fenotipos bioquímicos predominantes en ambos tipos de aislados, lo que sugiere que existe una diseminación de estos clones en el medio hospitalario y también en las aves.

En este estudio se determinó la presencia de cuatro genes de virulencia; aceI, gelE, agg y efaA en cepas de E. faecalis. En ambos tipos de aislados (humanos y pollos) fue demostrada la presencia de estos genes, ya fuese en forma aislada o en combinaciones. El gen efaA fue encontrado en la totalidad de las cepas de E. faecalis y no se detectó en otras especies de Enterococcus, lo que parece indicar que este gen podría ser un marcador específico de E. faecalis.

Los determinantes de virulencia fueron encontrados en mayor proporción en las cepas clínicas que en las aisladas en pollos, lo que concuerda con otros trabajos ${ }^{26}$. Sin embargo, sólo se observó significancia estadística en la presencia de los genes aceI y gelE. Los hallazgos de nuestro estudio señalan que $57,7 \%$ de las cepas de E. faecalis aisladas en hospitales albergaban los genes aceI-gelE-agg-efaA juntos y también, que en estas cepas clínicas, incluían algunos fenotipos bioquímicos predominantes, con RAN a aminoglucósidos, lo que ha sido descrito también en trabajos similares ${ }^{27}$.

Por otro lado, una amplia variedad de genes de virulencia han sido detectados en $E$. faecalis aislados de fecas de pollos y no en otras especies de Enterococcus ${ }^{28}$, lo que concuerda con nuestro trabajo. Es importante señalar que estas cepas de $E$. faecalis aisladas en pollos, que albergan genes de virulencia, pueden también constituirse en una fuente de transmisión al ser humano.

Se concluye que clones de E. faecalis con RAN a aminoglucósidos se han aislados de muestras clínicas y de pollos en el norte de Chile y que en algunos de ellos, se encontraron presentes genes de virulencia. Sin embargo, es necesario realizar nuevos estudios con un mayor número de cepas de Enterococcus para establecer si este tipo de resistencia está asociado a los factores de virulencia.

\section{Resumen}

Antecedentes: Enterococcus spp. es una causa importante de infecciones nosocomiales, tanto en Chile como internacional. Se han descrito una serie de factores de virulencia en este microorganismo, que pueden, por ejemplo, aumentar su habilidad para colonizar. Enterococcus tiene capacidad de adquirir genes de resistencia, entre ellos la resistencia de alto nivel (RAN) a los antimicrobianos aminoglucósidos. Objetivo: Investigar la prevalencia de genes de virulencia en cepas de E. faecalis susceptibles y resistentes a aminoglucósidos. Material y Métodos: Un total de 80 cepas de $E$. faecalis aisladas de muestras clínicas (n: 52) y pollos (n: 28) se incluyeron en este estudio. La identificación se hizo por pruebas bioquímicas y se tipificaron por el sistema Phene-Plate ${ }^{\mathrm{MR}}$. La susceptibilidad a diferentes antimicrobianos fue realizada por test de dilución en agar. Los genes de virulencia aceI, agg, gelE y efaA fueron investigados por RPC múltiple. Resultados: Todas las cepas de E. faecalis fueron susceptibles a vancomicina y ampicilina. Un 13,5\% de las cepas clínicas presentaron resistencia de alto nivel a gentamicina y $9,6 \%$ a estreptomicina. La tipificación reveló una gran diversidad de fenotipos, pero se encontró un clon con 7 cepas de características similares. El gen efaA estaba presente en 
$100 \%$ de las cepas, gen aceI en 94,2 y $75 \%$, gen $\operatorname{agg} 73,1$ y $67,9 \%$ y gen gelE 57,5 y $28,6 \%$ de las cepas clínicas y de pollos, respectivamente. Seis cepas con resistencia de alto nivel a aminoglucósidos, que pertenecían a un mismo fenotipo exhibieron los genes efaA, aceI, agg y gelE juntos. Conclusiones: Cepas de E. faecalis que albergan genes de virulencia y con resistencia de alto nivel a aminoglucósidos fueron aisladas de muestras clínicas y de pollos en Antofagasta. Se requieren mayores estudios para establecer una asociación entre estos factores.

\section{Referencias bibliográficas}

1.- Willems R J, Bonten M J. Glycopeptideresistant enterococci: deciphering virulence, resistance and epidemicity. Curr Opin Infect Dis 2007; 20: $384-90$.

2.- Tendolkar P M, Baghdayan A S, Shankar N. Pathogenic enterococci: new developments in the 21st century. Cell Mol Life Sci 2003; 60: 2622-36.

3.- Rice L B, Hutton-Thomas R, Lakticova V, Helfand M S, Donskey C J. $\beta$-lactam antibiotics and gastrointestinal colonization with vancomycin-resistant enterococci. J Infect Dis 2004; 189: 1113-8.

4.- Juliet C. Estudio de susceptibilidad in vitro de Enterococcus spp. Rev Chilena Infectol 2002; 19: 111-5.

5.- Fariñas M C, Torres C. Enterococo un patógeno emergente en nuestros hospitales. Enferm Infecc Microbiol Clin 2007; 25: 500-2.

6.- Fica A, Jemenao M I, Bilbao P, Ruíz G, Sakurada A, Pérez de Arce E, et al. Emergencias de infecciones por Enterococcus sp resistentes a vancomicina en un hospital universitario en Chile. Rev Chilena Infectol 2007; 24: 462-71.

7.- Vankerckhoven V, Van Autgaerden T, Vael C, Lammens C, Chapelle S, Rossi R, et al. Development of a multiplex PCR for the detection of asa1, gelE, cylA, esp, and hyl genes in enterococci and survey for virulence determinants among European hospital isolates of Enterococcus faecium. J Clin Microbiol 2004; 42: 4473-9.

8.- Eaton T J, Gasson M J. Molecular screening of Enterococcus virulence determinants and potential for genetic exchange between food and medical isolates. Appl Environ Microbiol 2001; 67: 1628-35.

9.- Vergis E N, Nathan S, Joseph W C, Hayden M K, Syndman D R, Zervos, et al. Association between the presence of enterococcal virulence factors gelatinase, haemolysin and enterococcal surface protein and mortality among patients with bacteremia due to Enterococcus faecalis. Clin Infect Dis 2002; 35: 570-5.
10.- Tendolkar P M, Baghdayan A S, Shankar N Putative surface proteins encoded within a novel transferable locus confer a high-biofilm phenotype to Enterococcus faecalis. J Bacteriol 2006; 188: 2063-72.

11.- Heikens E, Bonten M J, Willems R J. Enterococcal surface protein Esp is important for biofilm formation of Enterococcus faecium E1162. J Bacteriol 2007; 189: 8233-40.

12.- Shankar N, Coburn P, Pillar C, Haas W, Gilmore M. Enterococcal cytolysin: activities and association with other virulence traits in a pathogenicity island. Int J Med Microbiol 200; 293: 609-18

13.- Nallapareddy S R, Singh K V, Duh R W, Weinstock G M, Murray B E. Diversity of ace, a gene encoding a microbial surface component recognizing adhesive matrix molecules, from different strains of Enterococcus faecalis and evidence for production of ace during human infections. Infect Immun 2000; 68: 5210-7.

14.- Silva J, Loyola P, Galleguillos J C, Rodríguez Y, Colque-Navarro N, Möllby R, et al. Prevalencia de Enterococcus spp resistentes a antibióticos en aguas servidas en el norte de Chile. Rev Med Chile 2005; 133 : 1201-10.

15.- Silva J, Asserella L, Rodríguez Y. Prevalencia de Enterococcus spp. resistentes a los antibióticos en hospitales del norte de Chile. Rev Chilena Infectol 2006; 133: 226-31.

16.- Manero A, Blanch A R. Identification of Enterococcus spp. with a biochemical key. Appl Environ Microbiol 1999; 65: 4425-30.

17.- Saeedi B, Tärnberg M, Gill H, Hällgren A, Jonasson J, Nilsson L E, et al. Phene Plate (PhP) biochemical fingerprinting. A screening method for epidemiological typing of enterococcal isolates. APMIS 2005; 113: 603-12.

18.- Clinical and Laboratory Standards Institute. Performance Standards for Antimicrobial Susceptibility Testing; $16^{\text {th }}$ Informational Supplement. CLSI/NCCLS M100-S16: Clinical and Laboratory Standards Institute; 2006.

19.- Torres C, Tenorio C, Portillo A, García M, Martínez C, Del Campo R, et al. Intestinal colonization by vanA- or vanB2-containing enterococcal isolates of healthy animals in
Spain. Microb Drug Resist 2003; 9 (Suppl 1):

S47-52.

20.- Ministerio de Salud de Chile. Circular 4/C28, 9 de Mayo de 2000.

21.- Fica A, Jemenao M I, Bilbao P, Ruiz G, Sakurada A, Pérez de Arce E, et al. Emergency of vancomycin-resistant Enterococcus infections in a teaching hospital in Chile. Rev Chilena Infectol 2007; 24: 462-71.

22.- Braun S, Fica A, Andighretti B, Porte L, Triantafilo V. Estudio de factores de riesgo para colonización por enterococos resistentes a vancomicina en el Hospital Militar de Santiago. Rev Chilena Infectol 2009; 26: 374-5.

23.- López M, Hormazábal J C, Maldonado A, Saavedra G, Baquero F, Silva J, et al. Clonal dissemination of Enterococcus faecalis ST201 and Enterococcus faecium CC17-ST64 containing Tn5382-vanB2 among 16 hospitals in Chile. Clin Microbiol Infect 2009; 15: 586-8

24.- Donabediam S M, Thal L A, Hershberger E, Perri M B, Chow J W, Barltett, et al. Molecular characterization of gentamicinresistant enterococci in the United States: evidence od spread from animals to humans through food. J Clin Microbiol 2003; 41: 1109-13.

25.- Del Campo R, Tenorio C, Rubio C, Castillo J, Torres C, Gómez-Lus R. Aminoglycoside modifying enzymes in high-level streptomycin and gentamicin resistant Enterococcus spp. in Spain. Int J Antimicrob Agents 2000; 15: 221-6.

26.- Iversen A, Kühn I, Rahman M, Franklin A, Burman LG, Olsson-Liljequist B, et al. Evidence for transmission between humans and the environment of a nosocomial strain of Enterococcus faecium. Environ Microbiol. 2004; 6: 55-9.

27.- Klibi N, Ben Slama K, Sáenz Y, Masmoudi A, Zanetti S, Sechi L A, et al. Detection of virulence factors in high-level gentamicinresistant Enterococcus faecalis and Enterococcus faecium isolates from a Tunisian hospital. Can J Microbiol 2007; 53: 372-9

28.- Poeta P, Costa D, Rodrigues J, Torres C. Detection of genes encoding virulence factors and bacteriocins in fecal enterococci of poultry in Portugal. Avian Dis 2006; 50: 64-8 